Neutron powder diffraction study of silver subfluoride

This content has been downloaded from IOPscience. Please scroll down to see the full text. 1989 J. Phys.: Condens. Matter 12569

(http://iopscience.iop.org/0953-8984/1/15/002)

View the table of contents for this issue, or go to the journal homepage for more

Download details:

IP Address: 138.73.1.36

This content was downloaded on 22/07/2015 at 08:41

Please note that terms and conditions apply. 


\title{
Neutron powder diffraction study of silver subfluoride
}

\author{
Arthur Williams \\ Low Alamos Neutron Scattering Center, Los Alamos National Laboratory, Los Alamos, \\ NM 87545, USA
}

Received 16 August 1988

\begin{abstract}
Neutron powder diffraction measurements have been made at a number of different temperatures from room temperature to $20 \mathrm{~K}$ on a sample of silver subfluoride. The anti-CdI $\mathrm{C}_{2}$ structure has been confirmed and no evidence of a crystallographic phase transformation was discovered. We report the first measurements of the thermal parameters, strain, grain size, thermal expansion coefficients and vacancy density in this peculiar compound.
\end{abstract}

\section{Introduction}

Shortly after the turn of the century a large number of sub-metal halides in which the metal appeared with half its normal valence continued to appear in the literature. Of all these materials, only the enigmatic $\mathrm{Ag}_{2} \mathrm{~F}$ has withstood the test of time, all others having been found under more careful investigation to be non-existent.

Silver subfluoride is a bright, brassy yellow material with a hexagonal crystal structure, consisting of close-packed layers in the sequence $\mathrm{Ag}-\mathrm{Ag}-\mathrm{F}-\mathrm{Ag}-\mathrm{Ag}-\mathrm{F}$. It can be prepared chemically [1] or electrolytically [2], but decomposes into Ag metal and AgF in the presence of moisture, ultraviolet light, or above $80^{\circ} \mathrm{C}$. The transport properties are very anisotropic, having a room-temperature resistivity parallel to the layers about two orders of magnitude less than that perpendicular to the layers [3]. While conduction parallel to the layers is clearly metallic, both semiconducting [3] and metallic [4] behaviour has been claimed for conduction along the $c$ axis. Although band structure calculations [5] support the latter, photo-emission experiments [6] suggest that semiconducting behaviour is more likely. Below $66 \mathrm{mK}$ the compound exhibits bulk superconductivity [7].

Only two structural studies have been made to date on this unusual compound, [8, 9] both with $\mathrm{x}$-rays and both concluding the anti-CdI $\mathrm{C}_{2}$ structure, space group $\mathrm{P} \overline{3} \mathrm{~m} 1$, shown in figure 1. For this structure, the two Ag atoms shown in the unit cell are in exactly equivalent positions. In view of this claim that all $\mathrm{Ag}$ atoms are identical, the radiographic studies by Bruno and Santoro [10] are of particular interest. In their first experiment they found that following the formation of $\mathrm{Ag}_{2} \mathrm{~F}$ from inactive $\mathrm{AgF}$ and radioactive ${ }^{110} \mathrm{Ag}$ metal and subsequent decomposition in $\mathrm{H}_{2} \mathrm{O}$, the resulting $\mathrm{Ag}$ metal and $\mathrm{AgF}$ had equal activities. This is what would be expected given the equivalence of the silver atoms in the crystal structure. However, in a second experiment, in which natural $\mathrm{Ag}_{2} \mathrm{~F}$ was allowed to exchange with radioactive ${ }^{110} \mathrm{AgNO}_{3}$ in an acetone solution, 


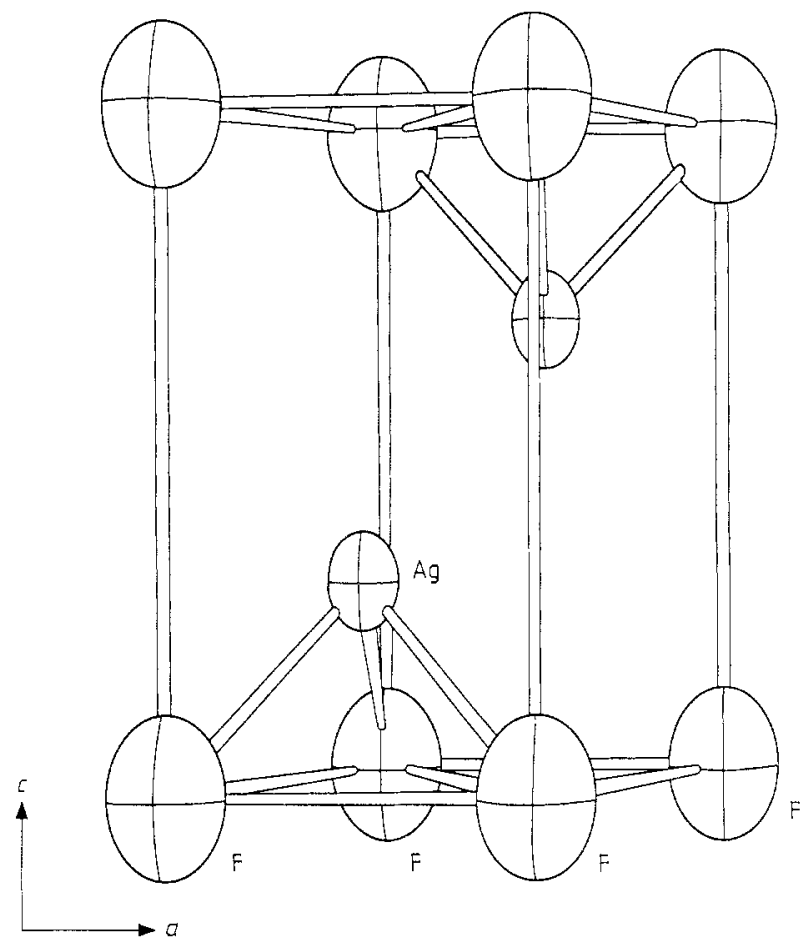

Figure 1. $\mathrm{Ag}_{2} \mathrm{~F}$ unit cell. The thermal ellipsoids are drawn as $99 \%$ probability surfaces for the refinement at $300 \mathrm{~K}$.

all the ${ }^{110} \mathrm{Ag}$ doped into the $\mathrm{Ag}_{2} \mathrm{~F}$ was recovered as $\mathrm{Ag}$ metal upon decomposition with $\mathrm{H}_{2} \mathrm{O}$. This latter result suggests the existence of two inequivalent crystallographic sites for $\mathrm{Ag}$ atoms in $\mathrm{Ag}_{2} \mathrm{~F}$, which is inconsistent with the proposed anti- $\mathrm{CdI}$ structure.

It is therefore of interest to make a more careful examination of the crystallography of silver subfluoride using modern neutron scattering and Rietveld refinement techniques. Using a pulsed spallation neutron source, the crystallographic parameters have been obtained over a wide temperature range. We also report the first thermal parameters, thermal expansion coefficients and estimate of the internal strain in chemically produced $\mathrm{Ag}_{2} \mathrm{~F}$ crystals.

\section{Experimental}

Silver subfluoride powder was prepared following the procedure described in [1]. The powder thus produced was carefully shielded from sources of ultraviolet light and protected from moisture in an inert-gas glove box equipped with a recirculating water vapour scrubber. Enough powder was made to completely fill a $9.5 \mathrm{~mm}$ diameter vanadium tube $50 \mathrm{~mm}$ long, which was sealed in dry helium gas with an indium O-ring seal. Neutron powder diffraction data was collected on the High Intensity Powder Diffractometer (HIPD), at the pulsed spallation neutron source at the Los Alamos Neutron Scattering Center (LANSCE). The sample tube was mounted to an Air Products recirculating ${ }^{4} \mathrm{He}$ Displex refrigerator and the temperature monitored and con- 


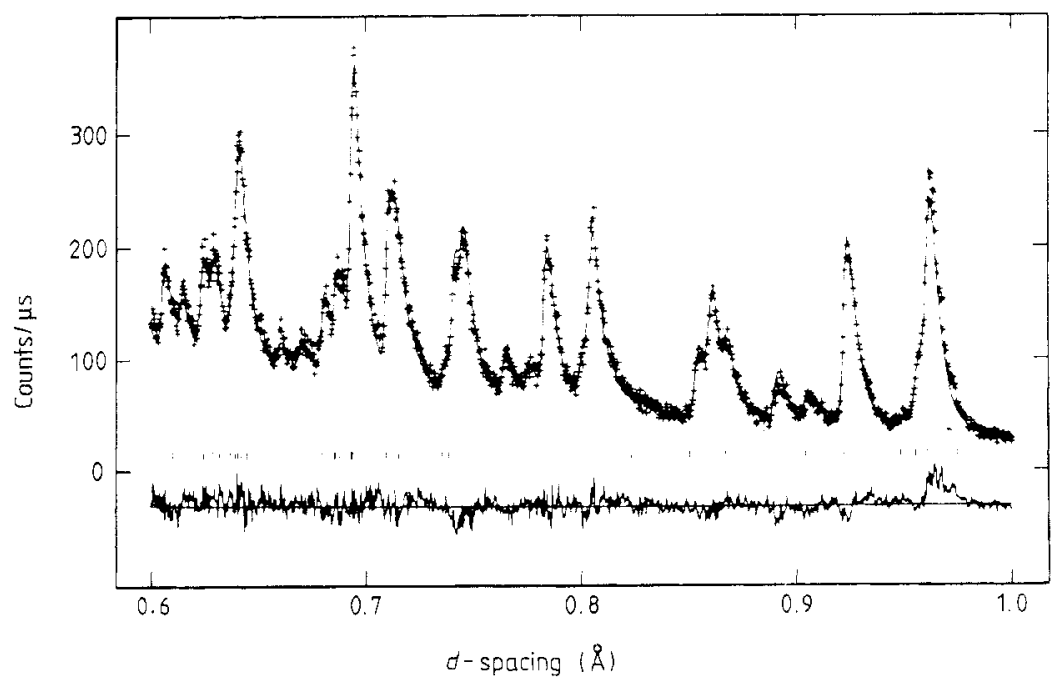

Figure 2. Partial neutron powder diffraction pattern of polycrystalline $\mathrm{Ag}_{2} \mathrm{~F}$ at $20 \mathrm{~K}$. The crosses are the data from the $+153^{\circ}$ detector bank and the full curve through the data is the Rietveld refinement best fit to the model. The tick marks show the positions of reflections allowed by the structure, and the full curve at the bottom is the difference between the data and the fit.

trolled by a Lakeshore DRC-81C PID controller. Diffraction data was collected in four detector banks, $\left( \pm 153^{\circ}\right.$ and $\left.\pm 90^{\circ}\right)$. The best resolution of the data obtained in these banks is about $\Delta d / d=0.3 \%$. The data acquisition program was set to hold at each temperature for one hour before beginning data collection in order for the rather massive sample to come to thermal equilibrium. Data collection times were about one hour for each temperature with LANSCE running at $805 \mathrm{MeV}$ and $20-30 \mu \mathrm{A}$ of proton current into the spallation target. The powder diffraction data was analysed using the General Structure Analysis System (GSAS) Rietveld refinement software package [11], using NBS silicon as an instrument calibration standard. The neutron powder peak profiles were modelled with the standard double-exponential-Gaussian convolution function $[12]$.

\section{Results}

Figure 2 shows part of a typical diffraction pattern obtained from the polycrystalline $\mathrm{Ag}_{2} \mathrm{~F}$ sample. Also shown are the Rietveld refinement fit to the data and the residual. The fits were to the anti-CdI ${ }_{2}$ structure and were in all cases found to be quite good with $R$-factors in the neighbourhood of 6 to $9 \%$ and reduced $\chi^{2}$ very close to unity. No evidence of any other phases or of any crystallographic phase transformations was found. Table 1 shows the crystallographic parameters obtained from the Rietveld refinements as well as the unit cell volumes and $c / a$ ratios at each of nine temperatures. The thermal expansion behaviour of this material is extremely anisotropic, with the linear expansion coefficient near room temperature in the $a b$ plane being about $2.8 \times 10^{-5} \mathrm{~K}^{-1}$, which is comparable to many metals, $\left(1.9 \times 10^{-5}\right.$ for $\mathrm{Ag}$ for example $)$. The linear expansion coefficient along the $c$ axis is four to five times smaller than that perpendicular to 
Table 1. Crystallographic parameters for silver subfiuoride obtained from Rietveld refinement to neutron powder diffraction data. The number in parentheses are the ESDs for the values. The cell volume and $c / a$ ratio are calculated from the values of the refined lattice parameters.

\begin{tabular}{llllll}
\hline$T(\mathrm{~K})$ & $a(\AA)$ & $c(\AA)$ & $c / a$ & Unit cell volume $\left(\AA^{3}\right)$ & $z$ \\
\hline 300 & $2.99877(5)$ & $5.6950(2)$ & $1.8991(1)$ & $44.351(3)$ & $0.3053(1)$ \\
250 & $2.99456(5)$ & $5.6932(2)$ & $1.9012(1)$ & $44.213(3)$ & $0.3050(1)$ \\
200 & $2.98955(4)$ & $5.6910(2)$ & $1.90363(8)$ & $44.049(2)$ & $0.3051(1)$ \\
150 & $2.98644(4)$ & $5.6897(2)$ & $1.90517(8)$ & $43.947(2)$ & $0.3049(1)$ \\
100 & $2.98280(4)$ & $5.6881(2)$ & $1.90697(8)$ & $43.828(2)$ & $0.3049(1)$ \\
50 & $2.97835(4)$ & $5.6877(2)$ & $1.90966(8)$ & $43.693(2)$ & $0.30481(9)$ \\
40 & $2.97735(4)$ & $5.6880(2)$ & $1.91043(8)$ & $43.667(2)$ & $0.30490(8)$ \\
30 & $2.97703(4)$ & $5.6881(2)$ & $1.91066(8)$ & $43.658(2)$ & $0.30501(9)$ \\
20 & $2.97685(4)$ & $5.6883(2)$ & $1.91088(8)$ & $43.654(2)$ & $0.30501(8)$ \\
\hline
\end{tabular}

the $c$ axis at room temperature and changes sign between $50 \mathrm{~K}$ and $100 \mathrm{~K}$, although the behaviour of both the unit cell volume and $c / a$ ratio is everywhere monotonic with temperature. The room-temperature volume thermal expansion coefficient, $(1 / V)(\partial T / \partial T)$ is about $6.9 \times 10^{-5} \mathrm{~K}^{-1}$, a value also typical of most metals and somewhat larger than that of silver, $\left(5.7 \times 10^{-5} \mathrm{~K}^{-1}\right)$. The atomic parameter, $z$, is nearly independent of temperature and has a value of 0.304 , in good agreement with the previous estimates from $\mathrm{x}$-ray diffraction work $[8,9]$.

The refinements of the peak profiles for this material exhibited no evidence for particle-size (Sherrer) broadening but a small amount of strain broadening. From the absence of any particle-size broadening and the limit of the resolution of the diffractometer, a lower limit of about $1000 \AA$ can be placed on the crystallite size of this sample. The refinements gave no evidence of extinction, from which an upper limit on the grain size of about $3 \mu \mathrm{m}$ can also be estimated. Refinement of the peak broadening contribution due to homogeneous isotropic strain in the sample consistently produced results between $0.2-0.3 \%$, with a weak tendency to decrease with decreasing temperature. Since no processing of the sample was done other than running the as-prepared powder through a 120 mesh sieve, this strain represents that which is 'grown in' the polycrystalline $\mathrm{Ag}_{2} \mathrm{~F}$ during its synthesis. Production of the material electrochemically or by a different chemical means may result in different values for the strain.

Table 2 shows the anisotropic thermal parameters obtained for each temperature. The thermal ellipsoids for both $\mathrm{Ag}$ and $\mathrm{F}$ atoms clearly exhibit much larger excursions along the $c$ axis than in the $a b$ planes by about a factor of two at room temperature and nearly a factor of five at $20 \mathrm{~K}$. The unit cell shown in figure 1 shows the actual thermal ellipsoids obtained from the refinement at $300 \mathrm{~K}$. From the very large values of $u_{33}$ for fluorine and its rapid increase with temperature it is not surprising that the compound decomposes at a relatively low temperature between 70 and $80^{\circ} \mathrm{C}$. At room temperature the $u_{33}$ value corresponds to an RMS displacement of the fluorine atom of $0.18 \AA$, more than $10 \%$ of the distance between adjacent Ag-F layers. Apparently the Ag-F bonding is very weak. By comparison, the ionic compound $\mathrm{AgF}$ is extremely stable, melting at $435^{\circ} \mathrm{C}$ and boiling at $1159^{\circ} \mathrm{C}$. The common claim $[4,6,9,13,14]$ that $\mathrm{Ag}_{2} \mathrm{~F}$ is an example of a mixed metallic-ionic bonded 'metal-salt' based on the similarity of its Ag-F bond length to that in $\mathrm{AgF}$ is clearly a serious over-simplification. $\mathrm{F}$ atoms in the subfluoride have three Ag atoms coordinated both above and below at $2.45 \AA$ and six coplanar F 
Table 2. Anisotropic thermal parameters for silver subfluoride obtained from Rietveld refinement to neutron powder diffraction data. The numbers in parentheses are the ESDS for the values.

\begin{tabular}{llllll}
\hline & \multicolumn{3}{c}{$F$} & \multicolumn{2}{c}{$\mathrm{Ag}$} \\
\cline { 2 - 3 } \cline { 5 - 6 }$T(\mathrm{~K})$ & $u_{11}, u_{22}\left(\AA^{2}\right)$ & $u_{33}\left(\AA^{2}\right)$ & & $u_{11}, u_{22}\left(\AA^{2}\right)$ & $u_{33}\left(\AA^{2}\right)$ \\
\hline 300 & $0.0164(5)$ & $0.0312(7)$ & & $0.0080(2)$ & $0.0160(3)$ \\
250 & $0.0133(4)$ & $0.0293(7)$ & & $0.0070(2)$ & $0.0146(3)$ \\
200 & $0.0100(3)$ & $0.0252(6)$ & & $0.0056(1)$ & $0.0119(2)$ \\
150 & $0.0078(3)$ & $0.0232(5)$ & & $0.0047(1)$ & $0.0105(2)$ \\
100 & $0.0060(3)$ & $0.0211(5)$ & $0.0036(1)$ & $0.0089(2)$ \\
50 & $0.0042(2)$ & $0.0182(5)$ & $0.00203(9)$ & $0.0073(2)$ \\
40 & $0.0036(2)$ & $0.0167(4)$ & & $0.00154(9)$ & $0.0068(2)$ \\
30 & $0.0033(2)$ & $0.0175(5)$ & $0.00154(9)$ & $0.0063(2)$ \\
20 & $0.0034(2)$ & $0.0166(4)$ & $0.00122(8)$ & $0.0061(2)$ \\
\hline
\end{tabular}

atoms in a hexagonal arrangement at 3.00 $\AA$. The fluorine in $\mathrm{AgF}$ ( $\mathrm{NaCl}$ structure) has only a symmetric octahedral coordination of six $\mathrm{Ag}$ atoms at $2.46 \AA$, each having one valence electron to donate to a fluorine to fill its outer shell, resulting in strong ionic bonds. The mixed-valent character of the electronic structure of silver in $\mathrm{Ag}_{2} \mathrm{~F}$ on the other hand results in a structure with much weaker covalent Ag-F bonds.

Ido and co-workers [4] have reported a Debye temperature of $120 \mathrm{~K}$ for $\mathrm{Ag}_{2} \mathrm{~F}$ from fits of the Bloch-Grüneisen formula to their electrical resistivity data. Resistivity measurements both parallel to and perpendicular to the $c$ axis of the single-crystal samples gave the same Debye temperature, suggesting that the scattering process is similar in both cases. From the temperature dependence of the thermal factors and the average atomic mass, it is also possible to make an estimate of the Debye temperature [15]. We obtain a value of $119 \mathrm{~K}$ from the values of $u_{x x}, u_{y y}$, in very good agreement with [4]. However, from the values of $u_{z z}$ we obtain a Debye temperature of $81 \mathrm{~K}$, indicative of the extremely anisotropic behaviour of this material. The resistivity, on the other hand, does not give a reliable indication of the vibrational anisotropy since it tends to weigh the higher energy phonons more strongly (which have a higher momentum to impart in a scattering event).

The density of $\mathrm{Ag}_{2} \mathrm{~F}$ has been measured by Terry and Diamond [9], who found that their experimentally obtained value, $8.64 \mathrm{~g} \mathrm{~cm}^{-3}$, was somewhat lower than that obtained from a calculation using the lattice parameters which they determined from $\mathrm{x}$ ray powder diffraction, $8.78 \mathrm{~g} \mathrm{~cm}^{-3}$. Hettich [2] also reports a rather low value for the measured density, in his case $8.57 \mathrm{~g} \mathrm{~cm}^{-3}$. In view of this we considered the possibility of the presence of defects in $\mathrm{Ag}_{2} \mathrm{~F}$ in the form of both $\mathrm{F}$ vacancies and $\mathrm{Ag}$ vacancies. We therefore performed Rietveld refinements of the occupations of both $\mathrm{F}$ and $\mathrm{Ag}$ at each temperature measured. No evidence of vacancies beyond the accuracy of the refined values of about one or two atomic per cent was observed. To account for the difference in measured and calculated densities reported by Terrey and Diamond [9] the compound would require an average stoichiometry of $\mathrm{Ag}_{2} \mathrm{~F}_{0.80}$ or $\mathrm{Ag}_{1.93} \mathrm{~F}$ for strictly $\mathrm{F}$ or $\mathrm{Ag}$ vacancies respectively. Even if all the vacancies were $\mathrm{Ag}$ our results are not adequate to explain the low measured densities of $\mathrm{Ag}_{2} \mathrm{~F}$ by other experimenters [2]. Since the accuracy of both lattice parameter and density measurements is ordinarily quite high, this discrepancy remains a mystery unless a high density of some other form of defect 
exists in the material or the density of the material is strongly dependent on the method of preparation.

\section{Conclusions}

We have confirmed the anti- $\mathrm{CdI}_{2}$ structure for $\mathrm{Ag}_{2} \mathrm{~F}$. In view of this the radiographic work of Bruno and Santoro [10] seems questionable. It is difficult to envisage a situation which would allow the complete separation of the ${ }^{110} \mathrm{Ag}$ doped into the single existing crystallographic site of $\mathrm{Ag}_{2} \mathrm{~F}$. No evidence for a structural phase transformaion was found between $20 \mathrm{~K}$ and $300 \mathrm{~K}$, although a pronounced minimum in the ' $c$ ' lattice parameter exists between $50 \mathrm{~K}$ and $100 \mathrm{~K}$. The polycrystalline sample prepared in this study was found to have about $0.2 \%$ internal strain at room temperature and a grain size between 0.1 and $3 \mu \mathrm{m}$. As with the electrical resistivity, the thermal expansion and vibrational behaviour of this material is very anisotropic. The expansion coefficient is much smaller along the $c$ axis than in the planes, while vibration amplitudes along the $c$ axis are much larger than the in-plane amplitudes. The $\mathrm{Ag}-\mathrm{F}$ bonding is much weaker than that found in AgF despite the similarity in their bond lengths. The claim by Pauling $[13]$ and others $[4,6,9,14]$ that $\mathrm{Ag}_{2} \mathrm{~F}$ is a rare example of mixed metallic and ionic bonding is incorrect. While metallic behavior certainly exists in the Ag planes, the Ag$\mathrm{F}$ bonding is intimately affected by the mixed-valent condition of the $\mathrm{Ag}$.

\section{Acknowledgment}

The author would like to thank Dr Andrew C Lawson for calling his attention to the existence of this fascinating layered material, as well as for many useful discussions.

\section{References}

[1] Won Choi Q 1960 J. Am. Chem. Soc. 822686

[2] Hettich A 1927 Z. Anorg. (Allg.) Chem. 16767

[3] Kawamura H, Shirotani I and Inokuchi H 1974 Chem. Phys. Lett. 24549

[4] Ido S, Uchida S, Kitazawa K and Tanaka S 1988 J. Phys. Soc. Japan 57997

[5] Hamada N, Ido S, Kitazawa K and Tanaka S 1986 J. Phys. C: Solid State Phys. 191355

[6] Sun F, Takahashi T, Takakuwa Y, Yaegashi H and Sagawa T 1986J. Phys. Soc. Japan 55461

[7] Andres K, Kuebler N A and Robin M B 1966 J. Phys. Chem. Solids 271747

[8] Ott H and Seyfarth H 1928 Z. Kristallogr. 67430

[9] Terrey H and Diamond H 1928 J. Chem. Soc. 2820

[10] Bruno M and Santoro V 1956 Gaz. Chim. Ital. 861095

[11] Larson A C and Von Dreele R B 1987 Los Alamos National Laboratory Report LA-UR-86-748

[12] Von Dreele R B, Jorgensen J D and Windsor C G 1982 J. Appl. Crystallogr. 15581

[13] Pauling L 1940 The Nature of the Chemical Bond 2nd edn (Ithaca, NY: Cornell University) p 421

[14] Trzebiatowski W and Rozyczka J 1958 Rocz. Chem. 32183

[15] Lawson A C and Williams A unpublished results 